\title{
UNA FÓRMULA CURIOSA
}

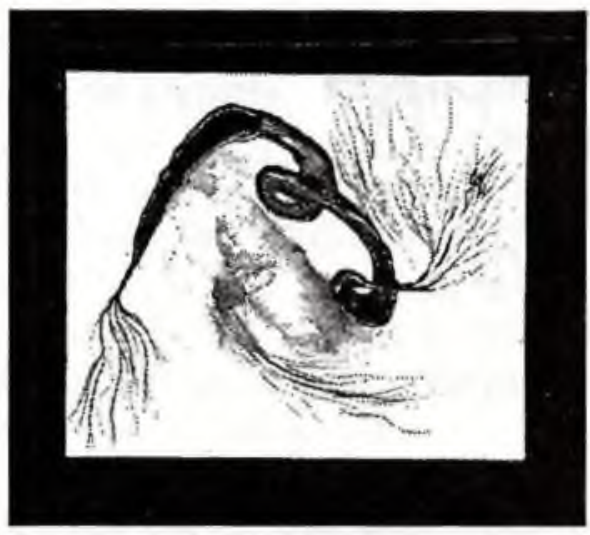

El objetivo de este artículo es mostrar una fórmula que permita calcular derivadas de orden alto, digamos 4, 5 ó más, de un producto de funciones sin tener que conocer las derivadas intermedias. El resultado es curioso, dado que guarda mucha analogía con la fórmula conocida con el nombre de Teorema del Binomio:

$$
(a+b)^{n}=\sum_{k=0}^{n}\left(\begin{array}{l}
n \\
k
\end{array}\right) a^{n-k} b^{k}
$$

En particular:

$(a+b)=a+b=a^{\prime} b^{a}+a^{a} b^{\prime}$

$(a+b)^{2}=a^{2}+2 a b+b^{2}=a^{2} b^{2}+2 a b+a \dot{u}$

$(a+b)^{3}=a^{3}+3 a^{2} b+3 a b^{2}+b^{3}=a^{3} b^{0}+3 a^{2} b+3 a b^{2}+a^{0} b^{3}$
Augusto Silva S.

Profesor Titular

Programa de Matemáticas y Física

Universidad Surcolombiana

Tratándose de derivadas vamos a convenir que la derivada cero de una función $f$ es la misma $f$, o sea $f^{(0)}=f$. Valen los resultados siguientes:

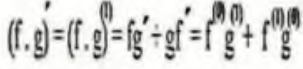

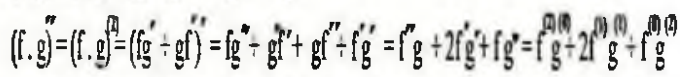

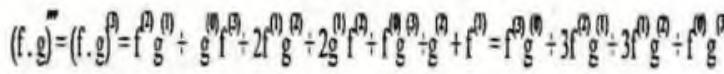

Obsérvense las analogías entre los coeficientes y los exponentes de $(a+b)^{1},(a+b)^{2},(a+b)^{3} y \quad$ las derivadas (f.g) (f.g) (f.g) ${ }^{\prime \prime}$. En general, se tiene el siguiente resultado : si $f, g$ son funciones que admiten derivadas hasta de orden $n$ entonces ( $f . g$ ) también admite derivadas de orden $\mathbf{n}$ y además

$$
(f . g)=\sum_{K=0}^{n}\left(\begin{array}{l}
n \\
k
\end{array}\right) f^{(n-k)} g^{(k)}
$$

Al igual que en el caso del Teorema del Binomio, este resultado también se prueba por inducción. En efecto:

1. Es claro que el resultado vale para $\mathrm{n}=1$.

2. Supongamos que el resultado vale para $n$ y veamos su validez para $n+1$ : 


$$
\begin{aligned}
& (f . g)^{(n+1)}=\sum_{k=0}^{D}\left(\begin{array}{l}
a \\
K
\end{array}\right)\left|\begin{array}{ll}
f^{(n-k)} & g^{(k)}
\end{array}\right|=\sum_{k=1}^{n}\left(\begin{array}{lll}
n \\
k
\end{array}\right)\left|\begin{array}{lll}
(n-k) & (k+1) & (n-k+1)
\end{array}\right| \\
& =\sum_{k=1}^{n}\left(\begin{array}{l}
n \\
k
\end{array}\right) f^{(n-1)(k+1)}+\sum_{k=0}^{n}\left(\begin{array}{l}
n \\
k
\end{array}\right) g^{(k)} f^{(n-k+1)} \\
& =\sum_{K=1}^{n+1}\left(\begin{array}{c}
0 \\
k-1
\end{array}\right) f^{(0-k+1)(k)} g^{(k)}+\sum_{k=0}^{n}\left(\begin{array}{l}
n \\
k
\end{array}\right) g^{(k)} f^{(n-k+1)} \\
& =\sum_{k=1}^{n}\left(\begin{array}{l}
n \\
k-1
\end{array}\right) f^{(n-k+1)(k)} g^{(n}+\left(\begin{array}{l}
(0) \\
k
\end{array}\right) g^{(n+1)}+\left(\begin{array}{l}
n \\
0
\end{array}\right) g^{(0)} f^{(n+1)}+\sum_{k=1}^{n}\left(\begin{array}{l}
n \\
k
\end{array}\right) g^{(k)} f^{(n-k+1)}
\end{aligned}
$$

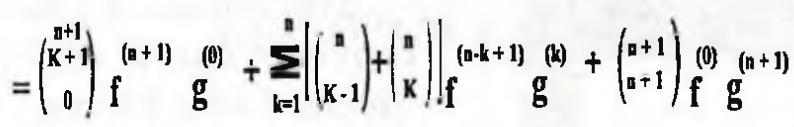

$$
\begin{aligned}
& =\left(\begin{array}{c}
K+1 \\
0
\end{array}\right) f^{(n+1)} \mathrm{g}^{(0)}+\sum_{k=1}^{0}\left(\begin{array}{c}
(n+1) \\
K
\end{array}\right) \mathrm{f}^{(0-k+1)} \mathrm{g}^{(\mathrm{k})}+\left(\begin{array}{l}
(\mathrm{n}+1 \\
(\mathrm{n}+1
\end{array}\right) \mathrm{f}^{(0)} \mathrm{g}^{(\mathrm{a}+1)} \\
& =\sum_{k=0}^{n+1}\left(\begin{array}{c}
n+1 \\
k
\end{array}\right) f^{(n-k+1)(k)}
\end{aligned}
$$

A manera de ejemplo, la cuarta derivada de $\mathrm{e}^{\mathrm{x}} \operatorname{sen} \mathrm{x}$ es :

M

A

$\mathrm{T}$

M

Á

$T$

I

C

A

S

$$
\left(e^{x} \operatorname{sen} x\right)^{(4)}=\left(e^{x}\right)^{(4)}(\operatorname{sen} x)^{(0)}+4\left(e^{x}\right)^{(3)}(\operatorname{sen} x)^{(1)}+6\left(e^{x}\right)^{(2)}(\operatorname{sen} x)^{(2)}+4\left(e^{x}\right)^{(1)}(\operatorname{sen} x)^{(3)}
$$$$
+\left(e^{x}\right)^{(0)}(\operatorname{sen} x)^{(4)}=e^{x} \operatorname{sen} x+4 e^{x} \cos x-6 e^{x} \operatorname{sen} x-4 e^{x} \cos x+e^{x} \operatorname{sen} x
$$$$
=2 e^{x} \operatorname{sen} x-6 e^{x} \operatorname{sen} x=-4 e^{x} \operatorname{sen} x
$$

\section{BIBLIOGRAFÍA}

1. APOSTOL, Tom. Calculus. Volumen I, Editorial Reverté, 1976

2. COURANT, R., Robbins, H., ¿Qué es la matemática?. Editorial Aguilar, 1976

3. RAINVILLE, Earl D., Ecuaciones diferenciales elementales. Editorial Trillas, 1979 